\title{
Batch-Fabricated, Addressable, Magnetically Actuated Microstructures
}

\author{
Jack W. Judy and Richard S. Muller \\ Berkeley Sensor \& Actuator Center (BSAC) \\ Department of EECS, University of California \\ Berkeley, California 94720-1770
}

\begin{abstract}
Surface-micromachined, batch-fabricated structures that combine plated-nickel films with polysilicon mechanical flexures to produce individually addressable, magnetically activated devices have been fabricated and tested. Individual microactuator control has been achieved two ways: (1) by actuating devices using the magnetic field generated by coils integrated around each device, (2) by using electrostatic forces to clamp selected devices to an insulated ground plane while unclamped devices are freely moved through large out-of-plane excursions by an off-chip magnetic field. The present application for these structures is as micromirrors for microphotonic systems where they can be used either for selection from an array of mirrors or else individually for switching among fiber paths.
\end{abstract}

\section{INTRODUCTION}

Magnetic actuation of polysilicon flexural MEMS elements provides several desirable features. Techniques to batch-fabricate these elements using electrodeposited NiFe films together with details of their performance were presented at this workshop in 1994 [1-2]. Based upon this earlier work, the following features were demonstrated.

(1) Large deflections ( $>1 \mathrm{~mm}$ ) are achieved using magnetic forces to actuate compliant microflexures.

(2) These large deflections can be achieved both parallel and perpendicular to the plane of the wafer.

(3) Actuation can be achieved using magnetic fields generated by either on- or off-chip sources (enabling remote device control)-- or by applying both at the same time.

(4) The actuating force can be applied in a conducting environment such as a saline fluid.

Torsion-beam suspensions of these magnetic elements were described in later work [3], that demonstrated their applicability for optical scanners, displays, and switches. We report here an enhancement of the static actuation model, a dynamic actuation model, and two methods for controlling individual flexure-based ferromagnetic microactuators. The first method uses coils integrated around each device to produce the magnetic field required for individual microactuator motion (Figure 1). The second method uses electrostatic forces to clamp selected devices to the insulated ground plane, while the off-chip magnetic field actuates unclamped devices (Fig. 2).

\section{STATIC ACTUATION}

A static mechanical and magnetic analysis of these torsional ferromagnetic microactuators has been given [3]. The accuracy of the model in reference [3] can be enhanced, particularly at low magnetic fields (where agreement with experimental results is least accurate), by accounting for the coercivity $H_{c}$ of the magnetic material. If $H_{\mathrm{c}}$ is considered, the magnetization $M$ in the film is expressed as

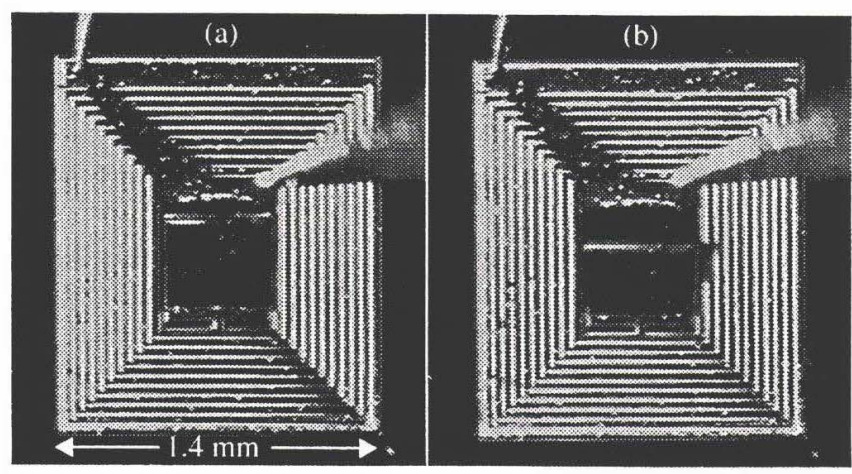

Figure 1. Top views of a microactuated torsion-beam element $(0.45$ mm square) surrounded by a 10-turn coil; a) no current applied to the coil, b) rotated $45^{\circ}$ by $H \approx 5 \mathrm{kA} / \mathrm{m}$ when $500 \mathrm{~mA}$ is applied to the coil.

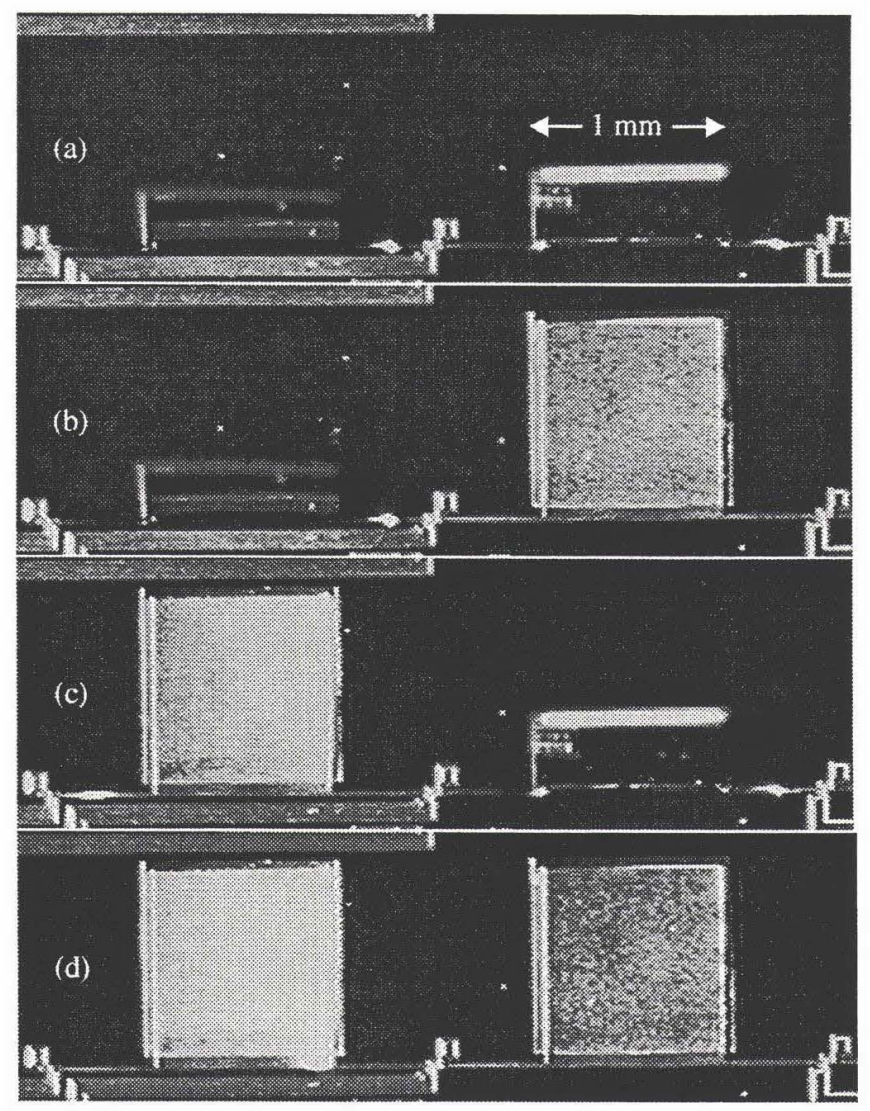

Figure 2. Top views of electrostatically addressed torsion-beam elements (1 mm square); (a) both devices actuated by an off-chip magnetic field $H \approx 8 \mathrm{kA} / \mathrm{m}$, (b) right device electrostatically clamped down and left device actuated, (c) left device clamped down and right device actuated, (d) both devices clamped down $\left(V_{c} \approx 5 V\right)$. 


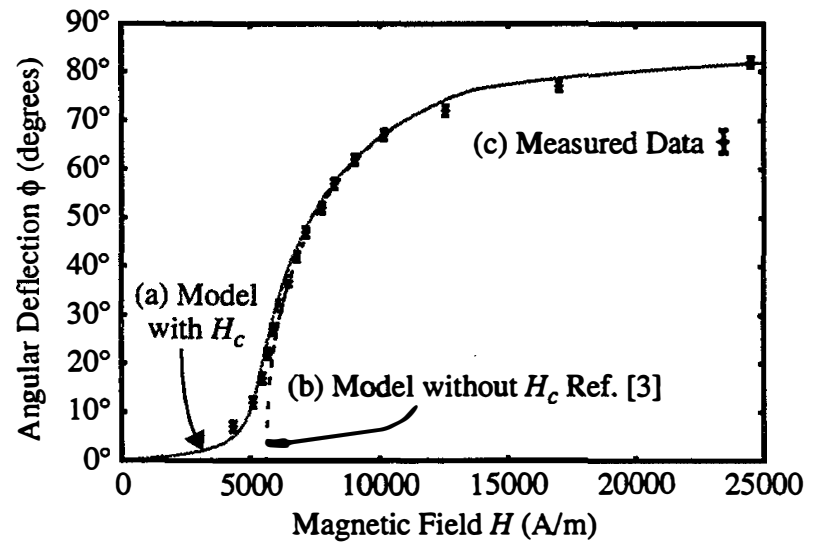

Figure 3. Comparison of calculated and measured dc-angular deflection as a function of magnetic field.

$$
M \approx \min \left(\frac{\mu_{o}\left(H_{c}+H_{e x t} \cos (\gamma-\theta-\phi)\right)}{\sqrt{N_{l}^{2} \cos ^{2} \theta+N_{t}^{2} \sin ^{2} \theta}}, \quad M_{s}\right)
$$

with the permeability of free space $\mu_{o}$, the angle between the external magnetic field and the substrate $\gamma$, the angular deflection of the magnetization from its preferred axis (the easy axis) $\theta$, the angular mechanical deflection from the substrate $\phi$, and the magnetic-shape coefficients along the length $N_{l}$ and thickness $N_{t}$ of the ferromagnetic element. Figure 3 shows the angular deflection predicted (a) by Eq. (1) (with $H_{c}=250 \mathrm{~A} / \mathrm{m}$ ), (b) by Eq. (12) of reference [3], and (c) the measured results. Equation (1) is seen to provide excellent agreement even at low magnetic fields.

\section{DYNAMIC ACTUATION}

To predict actuation under ac conditions, we formulate a dynamic torsional model:

$$
J \ddot{\phi}+C_{\phi} \dot{\phi}+k_{\phi} \phi=T_{m}
$$

with angular moment-of-inertia $\mathrm{J}$, angular damping coefficient $C_{\phi}$, angular stiffness of the torsional beam $k_{\phi}$, and driving magnetic torque $T_{m}$. The angular damping coefficient can be expressed as $C_{\phi}=\sqrt{J k_{\phi} / Q}$ with quality factor $Q$. Equation (2) is not a simple linear differential equation because the torque $T_{m}$ is a function of the angle between the ferromagnetic element and the substrate $\phi$. If we assume that the magnetization is along the easy axis $(\theta=0)$, and that the field is perpendicular to the substrate $\left(\gamma=90^{\circ}\right)$, the torque $T_{m}$ is

$$
T_{m}=V_{m a g} M\left(H_{d c}+H_{a c} \sin 2 \pi f t\right) \cos (\phi)
$$

with magnet volume $V_{\text {mag }}$, dc field $H_{d c}$, ac field $H_{a c}$, and drive frequency $f$. When Eq. (3) is substituted into Eq. (2), the result can be solved numerically. A plot comparing calculations for the mechanical frequency response with experimental results, is given in Fig. 4 for a device consisting of a $430 \times 130 \times 15 \mu \mathrm{m}^{3}$ NiFe plate $(J=$ $4.57 \times 10^{-16} \mathrm{~kg}-\mathrm{m}^{2}$ and $V_{\text {mag }}=8.4 \times 10^{-13} \mathrm{~m}^{3}$ ) attached to a pair of $400 \times 2.2 \times 2.2 \mu \mathrm{m}^{3}$ polysilicon torsional beams $\left(k_{\phi}=2 \mathrm{nN}-\mathrm{m} / \mathrm{rad}\right)$ with fitting parameter $Q=100$. Two features of the plot in Fig. 4 are readily apparent; first, the model agrees quite well with measurements, second, the peak of the resonance curve leans to the left, indicating that the system exhibits a nonlinear, hysteretic frequency response.

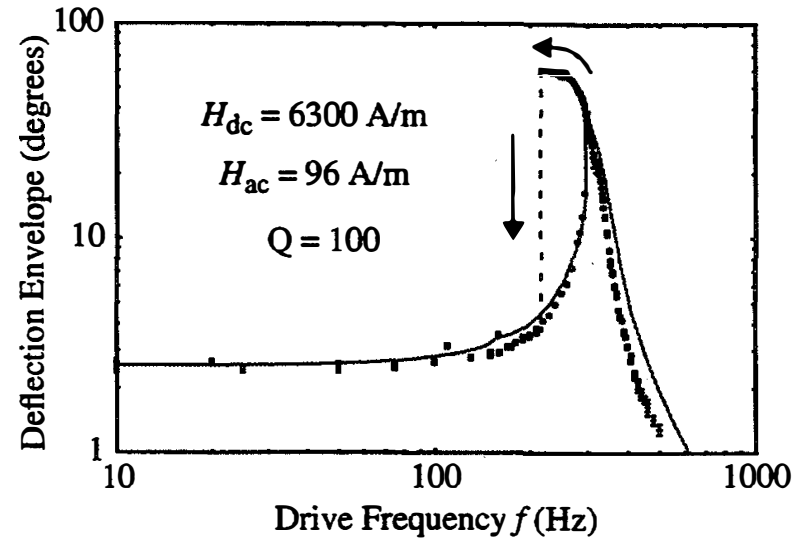

Figure 4. Comparison of calculated and measured mechanical frequency response.

\section{ADDRESSING}

A single off-chip field source can be used to actuate large arrays of microelements simultaneously, which can be useful for microtransport and microassembly applications [4], but actuation only of selected elements would permit a number of other uses. We have pursued two methods to achieve addressable actuation in an array of devices. The first method employs magnetic fields generated by coils integrated around each element to achieve a localized field and actuation (Fig. 1) [5]. An option for this scheme is to supplement this coil-driven field with an integrated ferromagnetic core. Although integrated coils with a ferromagnetic core can concentrate magnetic flux, thereby achieving a larger magnetic field, their fabrication process is considerably more complicated [6-7]. For our experiments, planar coils, which spiraled around each microactuator, were fabricated without any magnetic core. The second method for addressable actuation uses electrostatic forces to clamp selected devices to the insulated ground plane, so that the off-chip magnetic field does not affect the clamped devices (Fig. 2).

\section{INTEGRATED COIL}

Figure 1 shows a coil-driven device consisting of a $450 \mu \mathrm{m}$ square nickel pattern plated on polysilicon, which is attached to the insulated substrate via two torsional springs $200 \mu \mathrm{m}$ long, $2 \mu \mathrm{m}$ wide, and $2 \mu \mathrm{m}$ thick. The electroplated $5 \mu \mathrm{m}$-thick nickel is also used as the conducting layer for the 10-tum coil ( $25 \Omega$ ) surrounding the microactuator. The entire device, consisting of the coil and the moving structure, requires a square area $1.4 \mathrm{~mm}$ on a side. With no current in the coil, the device lays flat as in Fig 1a; when $500 \mathrm{~mA}$ is applied a field of $\sim 5 \mathrm{kA} / \mathrm{m}$ is generated that rotates the device more than $45^{\circ}$ out of the plane of the wafer. On-chip coil-actuated devices can achieve continuous positional control, however, high power $(6.25 \mathrm{~W})$ and large chip area $\left(2.25 \mathrm{~mm}^{2}\right)$ are required for this design.

Using copper instead of nickel for the coil would reduce the resistance by a factor of $\sim 4$ and also prevent the coil from shielding the device from any off-chip magnetic field, so that a small permanent magnet could be incorporated underneath the substrate to provide the dc field necessary to rotate the plate out of the plane of the wafer. Thus, the copper integrated coil would need to provide only a much smaller ac field. Driven at the resonant frequency, such a device should exhibit a large scanning envelope with significantly less power. 


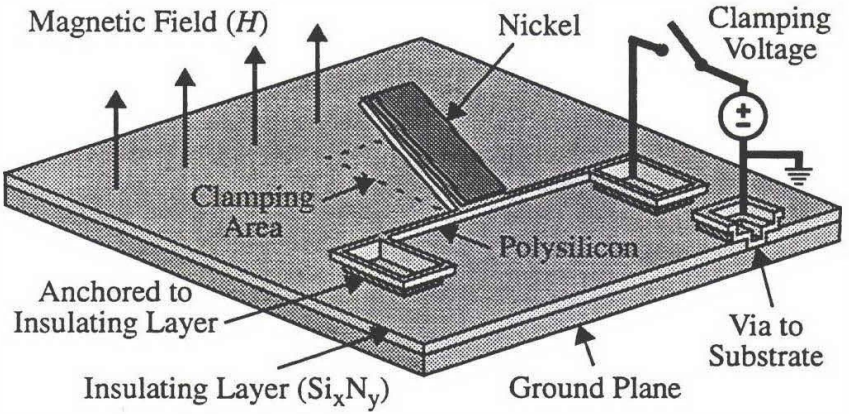

Figure 5. Schematic representation of the electrostatic clamping structure.

\section{ELECTROSTATIC CLAMP}

Individual selection of activated elements in an array, that is actuated by a uniform off-chip source, is possible using electrostatic forces to address individual microactuators, as is shown schematically in Fig. 5. Selection is accomplished by applying a voltage to the microactuator, which is anchored to an insulating dielectric layer, while the substrate is grounded. The resultant electrostatic force clamping the element to the surface, must be sufficient to keep selected devices from moving in response to the magnetic field applied to actuate unselected devices.

The clamping force $F_{c}$ is approximately that felt by the plates of a simple capacitor

$$
F_{c}=\frac{\varepsilon_{r} \varepsilon_{o} A V^{2}}{2 d^{2}}
$$

with relative permittivity of the dielectric layer $\varepsilon_{r}$ permittivity of free space $\varepsilon_{o}$, clamping area $A=w l$, voltage $V$, and electrode separation $d$. In Eq. 4 it is assumed that the clamping voltage is positive, putting the surface of the $n$-type silicon substrate into accumulation, and that trapped charge in the nitride and at the substrate-nitride interface is negligible. The clamping action can be formulated in terms of the clamping torque $T_{c}$, given by

$$
T_{c}=\int_{0}^{l} F_{c} d l^{\prime}=\frac{\varepsilon_{r} \varepsilon_{o} w l^{2} v^{2}}{4 d^{2}} .
$$

The clamping torque $T_{c}$ must exceed the drive torque $T_{m}$ for the clamp to be effective. If we assume that the external magnetic field is applied perpendicular to the substrate $\left(\gamma=90^{\circ}\right)$ and that the magnetization remains along the easy axis $(\theta=0)$, when the device is clamped $(\phi=0) T_{m}$ is

$$
T_{m}(\phi=0)=V_{\text {mag }} M H
$$

with magnet volume $V_{\text {mag }}=l w t$, magnetization $M$, and magnetic field $H$. The minimum clamping voltage $V_{c}$ derived by equating Eqs. (5) and (6) is

$$
V_{c}>\sqrt{\frac{4 M H t d^{2}}{\varepsilon_{r} \varepsilon_{o}^{l}}} .
$$

The electrostatic clamp has been experimentally investigated using the structures shown in Fig. 2. These devices consist of nickel-plated polysilicon plates ( $1 \mathrm{~mm}$ long, $1 \mathrm{~mm}$ wide, and $10 \mu \mathrm{m}$ thick) that rotate about a pair of torsional beams ( $400 \mu \mathrm{m}$ long, 15 $\mu \mathrm{m}$ (left device) to $20 \mu \mathrm{m}$ wide (right device), and $2 \mu \mathrm{m}$ thick) that are anchored to a $2.0 \mu \mathrm{m}$-thick silicon-nitride layer deposited on the n-type silicon substrate. For these plate structures, using $\left(\varepsilon_{r}=7.5\right.$ and $M=0.7 \mathrm{~T}$ ), a voltage of $V=4.1$ volts is calculated as sufficient to clamp the device in a field $H=10 \mathrm{kA} / \mathrm{m}$. Experimental results (a)

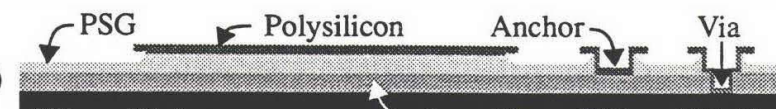

\section{Silicon Wafer} Low-Stress Silicon Nitride

(b)

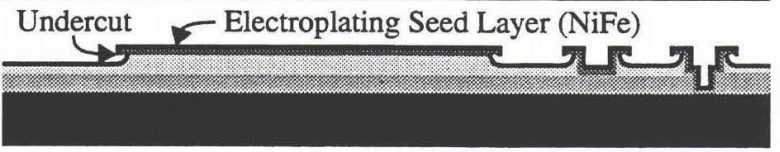

(c)

Photoresist Plating Mask

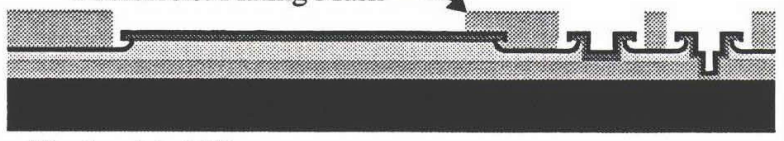

(d)
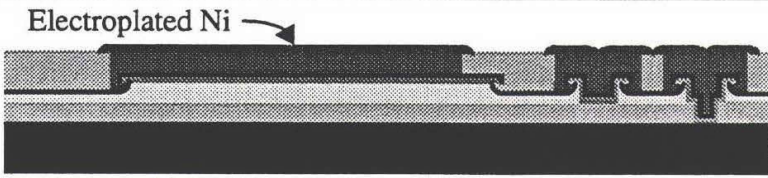

$$
\text { Sputter Etch }
$$

(e)

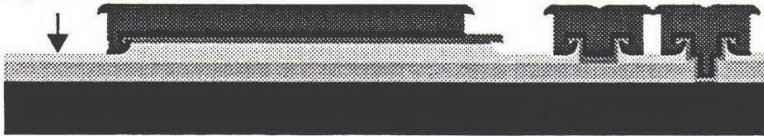

(f)

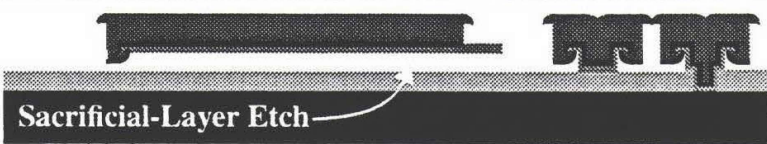

Figure 6. Fabrication process.

have confirmed that $\sim 5$ volts can clamp these structures in a $10 \mathrm{kA} / \mathrm{m}$ field.

The sequence of images shown in Fig. 2 illustrate individual device selection using electrostatic clamping. In Fig. 2(a), both microactuators are rotated up out of the plane of the wafer. The device on the right in Fig. 2a does not rotate as far as the device on the left because the beams supporting it are wider $(20 \mu \mathrm{m})$, and hence stiffer, than the beams supporting the device on the left (15 $\mu \mathrm{m})$. After taking the photograph in Fig. 2(a), the off-chip magnetic field has been turned off so that the devices retum to the substrate after which a clamping voltage is applied to the device on the right. Figure 2(b), taken after the magnetic field is tumed back on, shows the device on the right clamped down while the device on the left is rotated up by the magnetic field. In Fig. 2(c), the situation is reversed; that is, the device on the left is clamped down and the device on the right is rotated up by the field. Figure 2(d), shows the case of both devices clamped with the magnetic field applied.

Advantages of using electrostatic forces (instead of using integrated coils) to achieve individual microactuator control include the following:

(1) Arrays of elements can be readily addressed using wellknown digital-memory address techniques.

(2) The clamping scheme is easily incorporated in a batch-fabrication process.

(3) Clamping is accomplished with very little increase in the area of an array in conmast to that needed for on-chip coils.

(4) No static power is needed for the clamped devices.

\section{FABRICATION}

Both the coil-driven and the electrostatically addressed microactuators are constructed in a batch-fabrication process, as shown in Fig. 6, that combines ferromagnetic materials, nickel and nickel iron, and conventional silicon-process materials, single crystal-silicon, silicon nitride, phosphorus-doped silicon dioxide (PSG), 
and polycrystalline silicon (polysilicon). The ferromagnetic materials are introduced as a last step following nearly all the conventional IC processing.

The fabrication process begins with an n-type silicon wafer ( $\rho=10 \Omega-\mathrm{cm}$ ) which will be used as the ground plane. Although not done in this process, we could have, at this point, formed heavily-doped regions on the surface of the wafer to act as clamping counter electrodes. A $2.0 \mu \mathrm{m}$-thick layer of LPCVD siliconrich, low-stress silicon nitride is deposited on the wafer surface to form the insulating layer. The clamping voltage can be reduced by thinning the nitride layer, with the limit that it must be thick enough to survive the prolonged hydrofluoric acid (HF) release etch which slowly attacks silicon nitride. A $2.0 \mu$ m-thick layer of LPCVD PSG is deposited to form the sacrificial layer.

The first photolithography step defines holes through the PSG layer which will serve as insulated anchor positions for the microactuator. After stripping the first photoresist mask, a second lithography step defines holes through both the PSG and the silicon nitride layer so that electrical contact can be made to the substrate. After stripping the resist, a brief etch in HF is used to remove any native oxide and a $2.0 \mu \mathrm{m}$-thick layer of LPCVD polysilicon (605 ${ }^{\circ} \mathrm{C}$ ) is deposited to be used as the structural layer. After a $0.5 \mu \mathrm{m}$ thick layer of LPCVD PSG is deposited on top of the polysilicon, the wafer is annealed in nitrogen at $1000 \mathrm{C}$ for one hour to dope the polysilicon layer with phosphorus from the PSG and to reduce residual stress in the polysilicon film. A third lithography step defines the top PSG layer with the pattern for the mechanical structure. After the resist is stripped, the top layer of patterned PSG acts as a mask during a reactive-ion etch that selectively etches through the polysilicon layer until it stops on the PSG sacrificial layer. The top PSG layer is then removed in a wet HF etch that also attacks the revealed sacrificial layer and undercuts the polysilicon microstructure by about $0.5 \mu \mathrm{m}$ (Fig. $6 \mathrm{a}$ ).

A $0.1 \mu \mathrm{m}$-thick layer of $\mathrm{NiFe}$ is sputter deposited to form the electroplating seed layer. Since sputtering deposits material in many directions, some NiFe is deposited underneath the undercut polysilicon layer (Fig. 6b). The fourth and final lithography step defines a $4.0 \mu \mathrm{m}$-thick resist plating mask, which must be thick enough to cover the step from the sacrificial-PSG layer to the structural-polysilicon layer (Fig. c). If the mask is too thin, spurious hemispherical electrodeposits will form where the electric field breaks down the resist, particularly at the comers of the polysilicon structure where the electric field is concentrated. The plating mask is defined $50 \mu \mathrm{m}$ beyond the edge of the polysilicon plate on three edges (the far end and $90 \%$ of the way back along the sides toward the torsional bars). A significant overexposure is required to develop and remove the resist fully from underneath the polysilicon overhang.

Nickel is next electroplated onto the exposed areas of the seed layer, covering the top, the side, and underneath the polysilicon overhang (Fig. 6d). Thus, the ferromagnetic material is formed in a shape that essentially grasps the polysilicon layer and thereby achieves a good mechanical bond, which is helpful because nickel does not adhere well to polysilicon. We have found that adhesion layers, such as chrome and titanium, do not perform satisfactorily when exposed to a prolonged HF release etch. After the resist plating mask is stripped, the excess seed layer is removed by sputter etching (Fig. 6e), and the wafer is placed in a prolonged HF etch that removes the PSG sacrificial layer (Fig. 6f) (30 minutes for the devices shown in Fig. 2). A supercritical carbon dioxide drying technique is used to prevent the microstructures from sticking to the substrate after the release step [8].

\section{CONCLUSIONS}

We have shown that ferromagnetic microactuators with torsional-polysilicon flexures, capable of very large out-of-plane displacements (> $1 \mathrm{~mm}$ ), can be individually controlled with integrated coils or individually addressed by clamping with electrostatic forces. Individual prototype-torsional devices are shown to be deflected $45^{\circ}$ out of the plane of the wafer, by the magnetic field generated when a current of $500 \mathrm{~mA}$ flows through a 10-turn coil integrated around each device. Individual prototype-torsional devices, fabricated on a dielectric coated ground plane, can be electrostatically clamped in place using $5 \mathrm{~V}$ address potentials when an off-chip magnetic field of $10 \mathrm{kA} / \mathrm{m}$ is applied. This field torques unaddressed structures roughly into a perpendicular position. We have also shown that ferromagnetic films can be compatibly integrated with silicon-based materials and processes into a batch-fabrication process with good adhesion results using an under-etch technique to hold the films to the polysilicon layers. By accounting for the coercivity of the ferromagnetic material, the agreement of the static-deflection model with experimental results is improved, particularly at low magnetic fields. A model predicting the dynamic mechanical behavior of these devices has been developed which agrees quite well with experimentally measured frequency responses. An important application area for individually controllable ferromagnetic microactuators with polycrystalline silicon torsion-bar flexures is to microphotonic applications, such as optical scanners, displays, and switches.

\section{REFERENCES}

1. J. W. Judy, R. S. Muller, and H. H. Zappe, "Magnetic microactuation of polysilicon flexure structures," Tech. Dig. Solid-State Sensor and Actuator Workshop (Hilton Head '94), Hilton Head Island, SC (June 13-16, 1994), pp. 43-48.

http://www-bsac.eecs.berkeley.edu/archive/conference/hh1994/jjudy/

2. J. W. Judy, R. S. Muller, and H. H. Zappe, "Magnetic microactuation of polysilicon flexure structures," IEEE Journal of Microelectromechanical Systems, v. 4, no. 4, pp. 162-169, 1995.

http://www-bsac.eecs.berkeley.edu/archive/journal/jmems/jjudy/

3. J. W. Judy and R. S. Muller, "Magnetic microactuation of torsional polysilicon structures," 8th International Conference on Solid-State Sensors and Actuators Digest of Technical Papers (Transducers '95), Stockholm, Sweden, (June 25-29, 1995), v. 1, pp. 332-335.

http://www-bsac.eocs.berkeley.edu/archive/conference/trans1995/jjudy/

4. C. Liu, T. Tsao, Y.-C. Tai, W. Liu, P. Will, and C.-M. Ho, "A micromachined permalloy magnetic actuator array for micro robotics assembly systems," 8th International Conference on Solid-State Sensors and Actuators Digest of Technical Papers (Transducers '95), Stockholm, Sweden, (June 25-29, 1995), v. 1, pp. 328-331.

5. B. Wagner, W. Benecke, G. Engelmann, and J. Simon, "Microactuators with moving magnets for linear, torsional, or multiaxial motion," Sensors and Actuators A (Physical), v. A32, no. 1-3, pp. 598-603, 1992.

6. C. H. Ahn, Y. J. Kim, and M. G. Allen, "A planar variable reluctance magnetic micromotor with fully integrated stator and wrapped coils," Proc. IEEE Micro Electro Mechanical Syst. (MEMS '93), Fort Lauderdale, Florida, (February 7-10, 1993), pp. 1-6.

7. H. Guckel, T. R. Christenson, H. J. Skrobis, T. S. Jung, J. Klein, K. V. Hartojo, and I. Widjaja, "A first functional current excited planar rotational magnetic micromotor," Proc. IEEE Micro Electro Mechanical Syst. (MEMS 93), Fort Lauderdale, Florida, (February 7-10, 1993), pp. 7-11.

8. G. T. Mulhern, D. S. Soane, and R. T. Howe, "Supercritical Carbon Dioxide Drying of Microstructures," 7th International Conference on Solid-State Sensors and Actuators Digest of Technical Papers (Transducers '93), Yokohama, Japan, (June 7-10, 1993), pp. 296-299. 